\title{
Managing Smart Grid Information in the Cloud: Opportunities, Model, and Applications
}

\author{
Xi Fang*, Satyajayant Misra ${ }^{\dagger}$, Member, IEEE, Guoliang Xue*, Fellow, IEEE, and Dejun Yang* \\ ${ }^{*}$ Arizona State University, Tempe, AZ, USA \\ ${ }^{\dagger}$ New Mexico State University, Las Cruces, NM, USA
}

\begin{abstract}
Smart Grid (SG), regarded as the next generation electric grid, will use advanced power, communication, and information technologies to create an automated, intelligent, and widely distributed energy delivery network. In this article, we explore how Cloud Computing (CC), a next-generation computing paradigm, can be used for information management of the SG and present a novel SG information management paradigm, called Cloud Service based SG Information Management (CSSGIM). We analyze the benefits and opportunities from the perspectives of both the SG domain and the $\mathrm{CC}$ domain. We further propose a model for CSSGIM and present four motivating applications.
\end{abstract}

\section{INTRODUCTION}

Smart Grid (SG), an enhancement of the 20th century electrical grid, is regarded as a system that uses two-way communication and information technologies, and computational intelligence in an integrated fashion across electricity generation, transmission, distribution and consumption to achieve an electric system that is clean, secure, reliable, efficient, and sustainable [6]. The evolution of the SG heavily relies on the utilization and integration of modern information technologies. With the development of new applications and services that can leverage the capability upgrades enabled by the advanced information system, the grid will keep becoming "smarter." However, the overwhelming heterogenous information generated in the SG due to widely deployed monitoring, metering, measurement, and control devices calls for a powerful and cost-effective information management paradigm for data processing, analysis, and storage.

Therefore, we suggest that the information technology (IT) industry should be involved to assist in the information management of the SG. More specifically, we explore how Cloud Computing (CC), a next-generation computing paradigm, serves the information management in the SG. The concept of CC is based on large data centers with massive computation and storage capacities operated by Cloud providers, which deliver computing as a service. Shared resources, software, information, and storage are provided to computers and other devices as a utility over a network. An SG information management paradigm, in which the management is (partially) accomplished via CC, is called Cloud Service based SG Information Management (CSSGIM).

Recently, researchers have studied how to use CC to help manage the SG. Simmhan et al. [11] analyzed the benefit

This research was supported in part by ARO grant W911NF-09-1-0467. The information reported here does not reflect the position or the policy of the federal government. of using Cloud platform for demand response optimization in the SG. Rusitschka et al. [10] presented a model for the SG data management based on $\mathrm{CC}$, which takes advantage of distributed data management for real-time data gathering, parallel processing for real-time information retrieval, and ubiquitous access. Nagothu et al. [8] proposed to use CC data centers as the central communication and optimization infrastructure supporting a cognitive radio network of smart meters. Nikolopoulos et al. [9] presented a decision-support system and a CC software methodology that bring together energy consultants, consumers, energy service procedures and modern web interoperable technologies. Kim et al. [7] proposed a Cloud-based demand response architecture for fast response time in large scale deployments. Our work advances this line of research by minutely analyzing the benefits and opportunities from the perspectives of both the SG domain and the $\mathrm{CC}$ domain, further proposing a model connecting these two domains, and presenting some motivating applications.

This article is structured as follows. We first analyze the benefits of using CSSGIM in Section 2. We then describe the model of CSSGIM in Section 3, and present four application scenarios in Section 4. In Section 5, we conclude this article and present some future research topics.

\section{BenEFITS AND OPPORTUNITIES}

In this section, we will analyze the benefits of CSSGIM and the opportunities from the perspectives of both the SG domain and the CC domain.

\section{A. The Smart Grid Domain}

First, CSSGIM can improve the level of integration and utilization of information in the SG. In many cases, autonomous business activities lead to "islands of information," and as a result the information in each department of electric utility is not easily accessible by applications in other organizations or departments [6]. However, ensuring that the information is widely available, with privacy enforced, is critical to the operations in the SG. If all or most of the information is stored and managed by a service provider in the Cloud, we actually have a fairly cost-effective way to integrate these islands of information. Furthermore, a high level information integration also provides potentials to increase the information utilization level and improve the quality of decisions in operations.

Second, CSSGIM can help simplify the information system design in the SG domain and reduce the operational cost. Some information management services (e.g. information storage, 
data analysis, and strategy computation) can be outsourced to the Cloud, and the electric utilities can request the service(s) and application(s) when needed. This makes sense for the three reasons in the following.

The first reason is that the SG is a complex system of systems, resulting in complicated interactions among energy, information, and communication subsystems [6]. Traditional electric utilities may not have enough expertise on the design and deployment of complicated information systems. Therefore, the evolution of the SG may require the more experienced IT industry to be involved.

The second reason is that once an information management service is provided as a utility by the Cloud, many electric utilities may use this service to realize some functions without designing and implementing their own services from scratch. Also, Cloud service providers will take care of service updates, upgrades, and optimizations. This improves the utilization of services and resources while reducing the operational cost of the actors in the SG domain, since they do not have to invest a large amount of money into complex and costly information system design, deployment, maintenance, and upgrade.

The third reason is that the resource demands for many computation and data intensive applications in the SG vary so much that a scalable platform is required to deploy and operate. For example, the resource demands for the utility vary over the time of the day, with peak operation occurring during the day and information processing needs slowing down at night [12]. The essential property of the CC - high scalability and on-demand - makes the scaling problem in the SG much easier to solve.

Third, CSSGIM can generate opportunities for the prosperity of the ecosystem of the SG. The technology (e.g. distributed generations [6]) enabling individuals and small businesses with the capability of generating electricity lowers entry barriers for new players and liberalizes the markets in the SG domain, since the electricity generated from the end users can be traded. CSSGIM further lowers the entry barriers, since it would be much more cost-effective for end users to use pay-as-you-go and on-demand $\mathrm{CC}$ to realize information management, compared with the case where they design and implement their own information systems. Furthermore, at present $\mathrm{CC}$ is one of the hottest areas of research where new exciting services and applications keep emerging. Various applications developed and deployed by the service providers offer a range of value added services to the consumers. This trend may also inspire new exciting information management services in the SG domain, which further advance the prosperity of the ecosystem of the SG.

\section{B. The Cloud Computing Domain}

The emerging $\mathrm{SG}$ is also a good opportunity to advance the CC industry. The SG is widely envisioned as a promising solution to the next-generation power system, which is expected to generate billions of dollars in revenue each year. Fortunately, the SG itself is an advanced platform with energy, communication, and information integrated, which provides a point of penetration for the IT companies to get involved in this new industry. The $\mathrm{CC}$ has achieved success in many areas, such as mobile business, electronic commerce, and data storage service. It should also take advantage of this opportunity to broaden the success. Note that some pioneer IT companies have launched some preliminary programs already. Microsoft Hohm [2] is an online web application that enables customers to analyze their energy usage and provides energysaving recommendations. Google launched PowerMeter [1] as a free energy monitoring service, providing visualizations of customer energy usage, the ability to share information with others, and personalized recommendations to save energy.

\section{A Model of Cloud Service Based Smart Grid INFORMATION MANAGEMENT}

In this section, we present a model of CSSGIM, shown in Fig. 1, which consists of the SG domain and the CC domain. The SG domain is composed of seven sub-domains, which are compliant with the standards defined by the National Institute of Standards and Technology (NIST) [3]. The CC domain is composed of three sub-domains. In the following, we first describe each domain and the corresponding sub-domains, then present how these two domains are connected, and finally discuss a model to enforce protection policy.

\section{A. The Smart Grid Domain}

According to the model of NIST [3], an SG consists of seven sub-domains: bulk generation, transmission, distribution, customers, markets, operations, and service providers, as shown in Fig.2. Let us first look at bulk generation, transmission, distribution, and customers.

A power grid basically works as follows. Usually, electricity is generated at a few central power plants by electromechanical generators. The generating plants are quite large and located away from heavily populated areas. The generated electric power is stepped up to a higher voltage for transmission in the transmission system. The transmission system moves the power over long distances to substations. Upon arrival at a substation, the power is stepped down from the transmission level voltage to a distribution level voltage. As the power exits the substation, it enters the distribution system. Finally, upon arrival at the service location, the power is stepped down again from the distribution voltage to the required service voltage(s). This process covers the basic idea of the bulk generation, transmission, distribution, and customer sub-domains.

Bulk Generation: The actors in this sub-domain are responsible for the bulk generation of electricity and the corresponding control, measurement, protection, and recording procedures. Therefore, the information management part in this sub-domain should 1) record key performance and quality of service issues such as scarcity (especially for wind and solar) and generator failures; 2) utilize the data provided by the market subdomain to schedule generation and simultaneously provide availability data to the markets; and 3) record the history of device operations and maintenance, and analyze the performance and the life expectancy of devices.

Transmission: The transmission system is the carrier of the bulk electricity over long distances. The information management part in this subdomain should provide monitoring, 


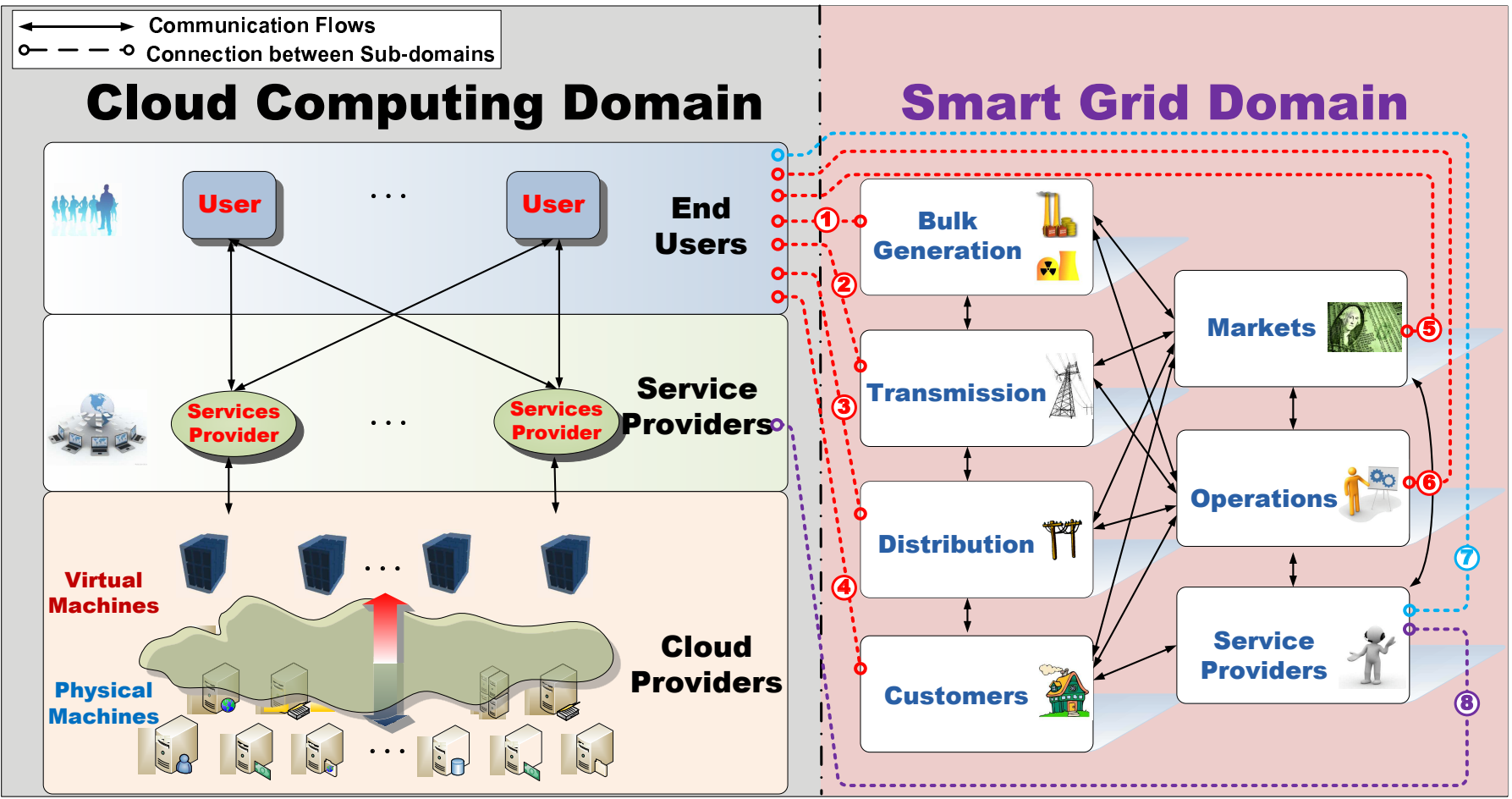

Fig. 1. Proposed Model of Cloud Service Based Smart Grid Information Management

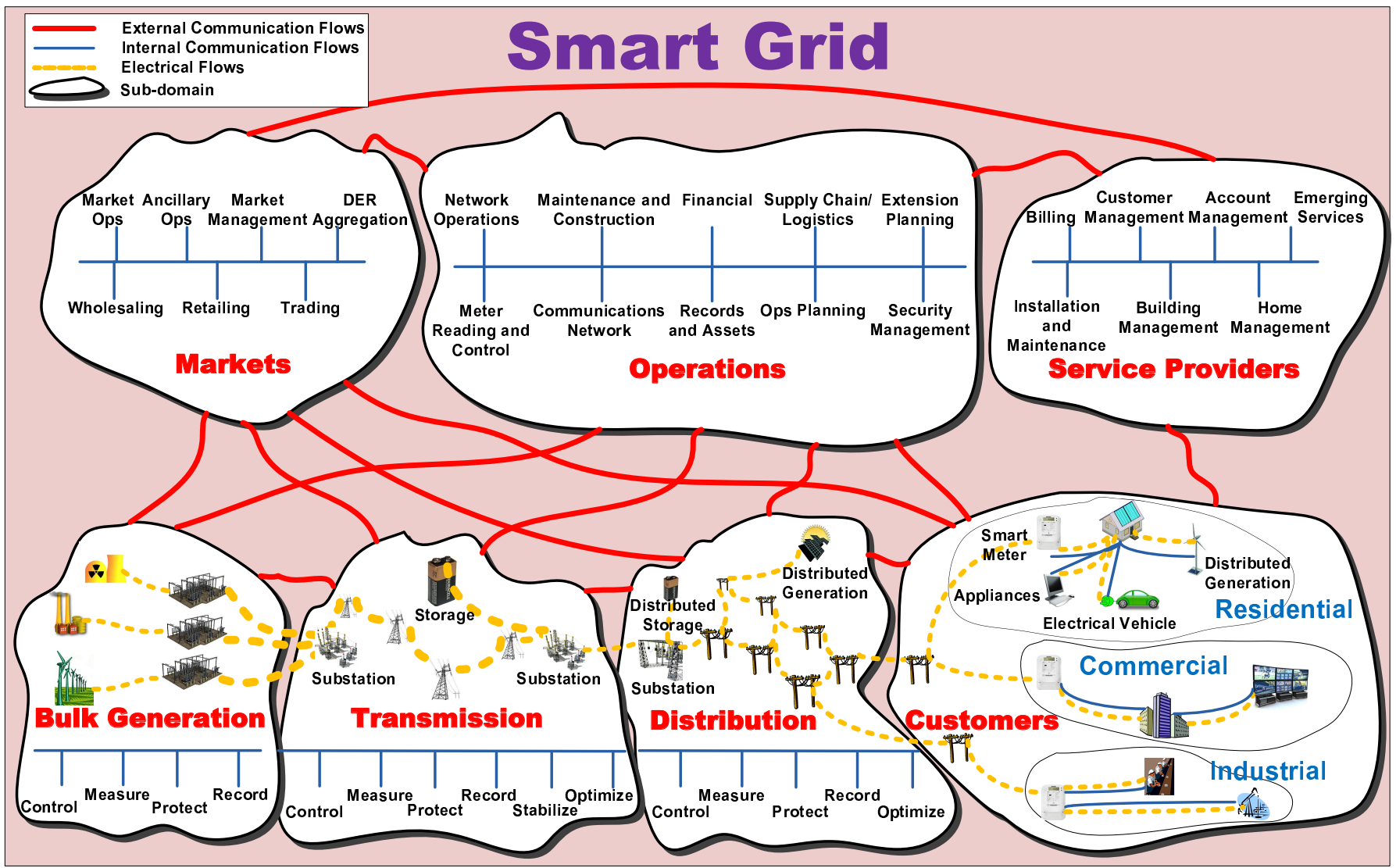

Fig. 2. Smart Grid Domain: For more details of each sub-domain, refer to the NIST report [3] 
information exchange, and control data for operations and control of transmission substations and field devices. This information is generated from widely-deployed measurement and monitoring devices, such as sensors and phasor measurement units [6]. Furthermore, this information should be properly used to manage the operations in the transmission system, including optimizing power flows, improving reliability, and optimizing asset utilization.

Distribution: The distribution system is the electrical interconnection between the transmission system and the customer sub-domain. As pointed out by NIST [3], the SG distribution system will communicate closely with the operation subdomain in real-time to manage the power flows associated with a more dynamic market sub-domain, and hence promptly adjust localized consumption and generation. A large amount of monitoring and control information, including load management and distribution system reliability, should be managed in this sub-domain.

Customers: The customer is ultimately the stakeholder that the entire power grid system is created to support. Customers are allowed to manage their energy usage, generation, and storage. The communication and information systems support the realization of many advanced user features, such as remote control, monitoring and control of distributed generation, inhome display of customer usage, automatic reading of meters, and control of new electric devices (e.g. electric vehicles).

In addition to the four basic sub-domains above, there are three other sub-domains supporting the SG: markets, service providers, and operations.

Markets: The market is the place where grid assets are bought and sold. The information management part of this sub-domain provides information support for the analysis and optimization of the pricing, for the balance of supply and demand, and for the energy trading between bulk generators, utilities, transmission operators, and customers.

Service Providers: The actors in this sub-domain perform services to support the business processes of other actors existing in the grid system. Such services may be performed by the electric service providers, existing third parties, or new participants drawn by the new business models. These business processes range from traditional services such as customer account management and billing, to enhanced new customer services such as management of energy usage and home energy generation [3]. Almost all of these processes need the involvement of the information management system, which 1) manages customer information; 2) utilizes usage data and pricing to encourage load control by the customer; 3 ) provides an effective information interaction interface between the customers and the markets; and 4) provides information support for the emerging services.

Operations: The actors in this sub-domain are responsible for the operation of the power system and the management of the movement of electricity. In order to maintain reliable and safe operation of the grid, the information management part in this sub-domain should support aggregation, validation, editing, estimation, and analysis of the monitoring, measurement, metering, and operation data gathered from the power grid.

\section{B. The Cloud Computing Domain}

In this subsection, we describe the three sub-domains of the CC domain in our model: Cloud providers, service providers, and end users.

Cloud Providers: A Cloud provider, the owner of the infrastructure, is responsible for managing physical and virtual resources to host applications and services, such as computing, data storage and management, and user developed applications. A Cloud provider has to assign virtual resources to be hosted on its physical resources [5] and can hence provide maximum flexibility to configure various configurations/partitions of physical resources to serve requests with differing requirements [4].

Service Providers: A service provider leases resources in the Cloud infrastructure provided by the Cloud providers and provides applications or services that will be utilized by end users. In order to differentiate a service provider in the CC domain from a service provider in the SG domain, we call the former one a CC service provider and the latter one an $S G$ service provider. In addition, some CC service providers may form a special class of actors, called service brokers. A service broker, as an actor with the expertise on the Cloud, mediates between end users and other $\mathrm{CC}$ service providers for gathering end user requirements, locating the best suitable CC service providers, and assisting end users in (dynamically) buying, obtaining, and releasing Cloud services [4]. The idea of service broker is compelling because as the number of services supported increases, end users may have more difficulties in finding a $\mathrm{CC}$ service provider that best meets their requirements, such as cost, availability, performance, and service category.

End Users: An end user generates demands for the Cloud to process and is the consumer of the services provided.

\section{Connection between the Smart Grid Domain and the Cloud Computing Domain}

In our model, we mainly use $\mathrm{CC}$ to assist information management tasks in the SG, such as storage, integration, validation, analysis, and optimization of various data and information existing in the SG domain. These tasks can be outsourced and offered by the CC domain as pay-as-you-go and on-demand services.

The actors in the information management part of the six SG sub-domains in the following - bulk generation, transmission, distribution, customer, market, and operation domains - work as the end users in the CC domain. The connections are shown by Lines 1-6 in Fig.1. These actors analyze their information requirements and ask for solutions in the $\mathrm{CC}$ domain as end users. In other words, these actors mainly focus on collecting and abstracting information service requirements from the SG domain without considering the details of how such services are fulfilled. The actors in the CC domain are responsible for the development, deployment, maintenance, optimization, and upgrade of such services.

The services or applications requested by the actors in the SG domain may already exist, such as large amount of information storage service. If such services or applications 
do not exist (e.g. user billing, account analysis, or grid status analysis), the $\mathrm{CC}$ service providers may need to design and deploy these new services. Therefore, many actors in the SG domain (e.g. electric utilities) may utilize these services to realize their functions. This actually improves the utilization of services and resources since different actors in the SG domain do not have to design and implement the same function from scratch. Moreover, the cost of update, upgrade, and maintenance can also be cut down, since the CC service providers will take care of these tasks.

The SG service providers may work as end users or even $C C$ service providers. The connections are shown by Lines 7-8 in Fig. 1. If SG service providers only abstract service requirements and ask the $\mathrm{CC}$ service providers to realize this service, SG service providers actually work as end users in the $\mathrm{CC}$ domain. However, sometimes SG service providers may also work as CC service providers. This is because the SG service providers usually understand the electric power industry better than traditional $\mathrm{CC}$ service providers. Therefore, it might be better if the SG service providers design the corresponding applications and services by themselves using the Cloud architecture. SG service providers may also specifically work as service brokers in the CC domain. As mentioned before, as the number of new services supported in the $\mathrm{CC}$ domain increases, end users may have more difficulties in finding the most suitable one that meets their requirements. Since the SG service providers have a good understanding of electric power industry, they, working as service brokers, should be more helpful to assist the end users in locating the required services if possible.

\section{Protection Policy}

Ensuring information security, privacy, and quality of service would be crucial to the CSSGIM. Information security and privacy may be the biggest concern in outsourcing information management to the Cloud [12]. Moreover, guaranteeing quality of service is important for grid operations. For example, failure to promptly diagnose the system failure from the operational data may lead to serious problems or even disastrous results [6]. We therefore propose a component called Protection Policy Manager (PPM), which resides in the SG domain but provides an interface between the actors in the SG domain and the actors in the $\mathrm{CC}$ domain to enforce security, privacy, quality of service, and other possible information management protection requirements. In practice, SG service providers can offer PPM as a service.

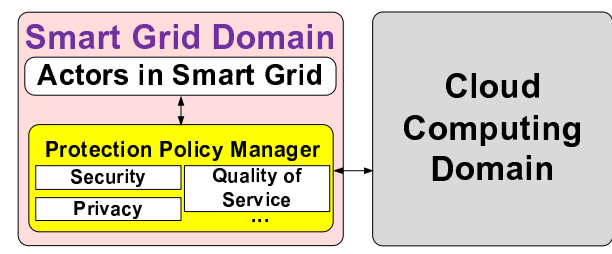

Fig. 3. Protection Policy Manager

The PPM has a set of strategies that are pre-computed or computed on the fly. It chooses appropriate strategies according to the protection requirements indicated by the actors in the SG. If no strategy that meets the requirements is found, the PPM then notifies the actors and lets them decide whether they are willing to decrease the protection levels. We list three possible strategies in the following. First let us look at the strategy of choosing trustworthy CC service providers. For example, for storing and processing critical and regulated information, the PPM chooses the private Cloud to fulfill the required information management tasks (as also suggested in [11]). In this way, data can be stored, processed, and transferred in a trustworthy and controlled environment. Second, let us look at the strategy of operating encryption on information storing and computation. For storing sensitive data, the PPM provides an extra layer of data encryption according to the requirements of the actors in SG. For processing sensitive data, the PPM protects sensitive information by enabling computations with encrypted data, validating the computation result, and recovering the required results from the output of the computations fed with encrypted data. Such secure computation outsourcing was recently shown to be feasible in theory and practice [13]. Third, let us look at the strategy of increasing the redundancy of information storing and computation. For storing a critical data item, the PPM may leverage storage virtualization technology. It increases the redundancy of this data item and stores different portions of this data item in different CC service providers. With careful redundancy design, if some portions of this data item are missing or compromised, we may still be able to recover the original data item. For some critical information computation tasks, the PPM may construct multiple copies and use different $\mathrm{CC}$ service providers to run them simultaneously. Once one of them is done on time, the service result can be delivered back to the actors in the SG domain.

\section{Applications}

In this section, we present four application scenarios that demonstrate the effectiveness of the SG - CC interplay.

\section{A. User Data Management and Application Service}

First, traditionally the user data is stored by, for example, the electric utility for billing information. However, in the emerging SG, user behavior is not always associated with the electric utility. For example, the SG emphasizes a grid paradigm - microgrid, which is a localized grouping of electricity generations, energy storages, and loads [6]. A microgrid can function autonomously without obtaining power from the electric utility. The operational and user data in a microgrid may not be stored in the electric utility because it functions autonomously. Using the Cloud to store and analyze this user data would be a good option. The user data storage and analysis services are provided by the CC service providers as "pay-as-you-go" services. The customers in the SG domain transfer the user data to the Cloud and request the corresponding services when needed.

Second, an important feature of the SG is that the use of two-way flows of information and energy enables many advanced applications or functions to control the operations of devices in the SG. One key role to realize this is the 
smart meter, which can obtain information from users' devices and appliances, while also controlling the behavior of the devices [6]. Many important functions, such as realtime user behavior analysis and demand response [6], are realized based on this platform. With the advancement of the SG, more and more functions may be invented and deployed. One important question is whether it is possible for us to add new applications to the platform, without the need to perform upgrades or hard resets. This issue may be solved with the use of a Cloud-Smart Meter Framework, as shown in Fig. 4.

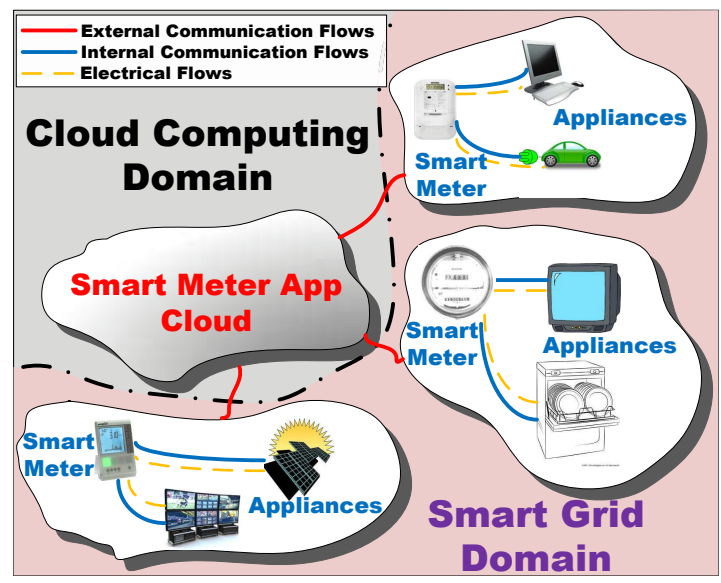

Fig. 4. Cloud-Smart Meter Framework

All the services are located in a Cloud, called Smart Meter App Cloud. The service development, maintenance, and update are accomplished by the service providers in this Cloud. A smart meter works as a command terminal, which requests services via a public interface, and controls the devices according to the information returned from the Cloud. This is analogous to the scenario where personal computers use a unified webbased interface (e.g. an Internet browser) to request and obtain Cloud services. Consider, for example, a smart air conditioner control service, which learns the human activity patterns in order to optimize the schedule of the air conditioner to balance the billing and warming/cooling. This service is located in the Smart Meter App Cloud. A smart meter, acting as an end user in the $\mathrm{CC}$ domain, requests this as a service and controls the air conditioner according to the information returned. If the machine learning algorithm in this service, which learns the human activity patterns, is optimized and updated, the smart meter just does the same thing (i.e. requesting the service and interpreting commands from the Cloud) without the need to know the update. In order to locate the suitable service providers in the Smart Meter App Cloud, the smart meter can ask the service broker for assistance.

\section{B. Advanced Trading Information Service}

In the emerging $\mathrm{SG}$, an advanced trading information service is needed to support more flexible power grid asset buying and selling operations. As mentioned before, distributed generation of electricity lowers the entry barriers for new players and liberalizes the markets in the SG domain, since personal users can trade their generated electricity. Therefore, the advanced infrastructure of the SG leads to many potential markets. Establishing and maintaining an advanced trading information system is probably costly, especially for small electric utilities or personal users who just want to do trading without the motivation to construct a trading system. Delivering a trading information service as a "pay-as-you-go" utility is hence compelling. The information storage, analysis, trading strategy computation, advanced trading options (e.g. auction), and even advertising are offered by $\mathrm{CC}$ service providers as services. The users provide the requirement of what they want to buy or sell, and request the service provider for a trading service. This is similar to somebody using an online stock trading program to trade stocks, bonds, etc., in a stock exchange. The trading information service system will find the match for the users.

\section{Grid Operation Information Analysis Service}

The emerging SG will generate a large amount of data, due to the wide-scale metering, sensing, and monitoring operations [6]. Storage, realtime analysis, and optimization of such large amount of data is a nontrivial task for traditional electric utilities. Outsoucing this task to the $\mathrm{CC}$ domain may be a compelling option, since the Cloud providers are usually equipped with a large number of massive data computing and storage centers. Therefore, electric utilities can transfer the data to the $\mathrm{CC}$ service providers and request storage, realtime analysis, and optimization of the data as services. Furthermore, once such information from multiple electric utilities is accessible and integrated by a CC service provider with data privacy rules enforced, the service provider may be able to provide better grid operation support based on more comprehensive information.

\section{Recommending, Consulting and Advertising Service}

The user energy usage data is full of user behavior information, which can be mined to provide energy recommendations or consultant advices for the users to help them reduce billing or help the grid operate better. This information can also be used to advertise appropriate energy saving appliances. From the perspective of product sellers, this information can improve the hit rate of advertising. An electric utility or a user can use such recommending, consulting and advertising services if they allow the $\mathrm{CC}$ service provider to access the user data with privacy rules enforced and then ask the service provider to deliver these services. This service can become smarter if more information (probably provided by other parties, such as location information providers and social network service providers) is integrated, with privacy enforced. For example, this service can leverage the user data from location information providers (e.g. Google Latitude) to provide an optimized electric vehicle charging scheduling for all the users it serves to avoid simultaneous charging, which will lead to a significant load on the existing distribution grids [6]. An illustrative example is shown in Fig. 5.

\section{Conclusions and Future Research}

In this article, we have analyzed the benefits and opportunities of using CC to help information management in the SG, 


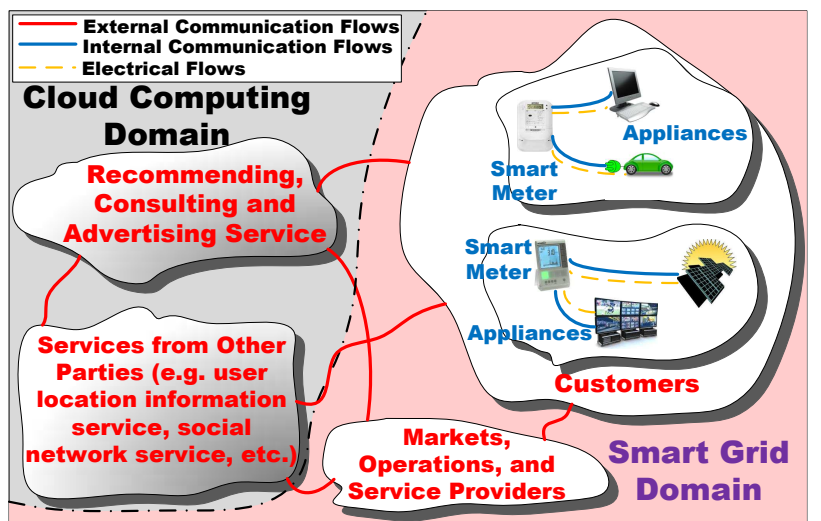

Fig. 5. Recommending, Consulting and Advertising Service

proposed a model to connect these two domains, and presented four application scenarios. Although there is a great promise to use $\mathrm{CC}$ to serve the information management in the SG, there are still many issues needing to be further solved to help this vision come true. For example, first it would be very important to assess potential security and privacy issues. Second, our model gets actors in the CC domain, the SG domain, and the intermediate communication provider domain involved and working together towards a common goal. Therefore, a systematic study of this multi-domain resource optimization problem is of great relevance not only for cost optimization, but also many other relevant parameters.

\section{REFERENCES}

[1] Google powermeter: http://www.google.com/powermeter.

[2] Microsfot hohm: http://www.microsoft-hohm.com.

[3] National Institute of Standards and Technology. NIST framework and roadmap for smart grid interoperability standards, release 1.0, http://www.nist.gov/public_affairs/releases/upload/smartgrid_ interoperability_final.pdf. January 2010.

[4] R. Buyyaa, C. S. Yeo, S. Venugopal, J. Broberg, and I. Brandic. Cloud computing and emerging it platforms: Vision, hype, and reality for delivering computing as the 5th utility. Future Generation Computer Systems, pages 599-616, 2009.

[5] P. Endo, A. de Almeida Palhares, N. Pereira, G. Goncalves, D. Sadok, J. Kelner, B. Melander, and J. Mangs. Resource allocation for distributed cloud: concepts and research challenges. IEEE Network, pages 42-46, 2011.

[6] X. Fang, S. Misra, G. Xue, and D. Yang. Smart grid - the new and improved power grid: A survey. IEEE Communications surveys and tutorial (Digital Object Identifier: 10.1109/SURV.2011.101911.00087).

[7] H. Kim, Y.-J. Kim, K. Yang, and M. Thottan. Cloud-based demand response for smart grid: Architecture and distributed algorithms. IEEE SmartGridComm'11, pages 398-403, 2011.

[8] K. Nagothu, B. Kelley, M. Jamshidi, and A. Rajaee. Persistent Net-AMI for microgrid infrastructure using cognitive radio on cloud data centers. IEEE System Journal (Digital Object Identifier: 10.1109/JSYST.2011.2162794).

[9] V. Nikolopoulos, G. Mpardis, I. Giannoukos, I. Lykourentzou, and V. Loumos. Web-based decision-support system methodology for smart provision of adaptive digital energy services over cloud technologies. IET Software, 5(5):454-465, 2011.

[10] S. Rusitschka, K. Eger, and C. Gerdes. Smart grid data cloud: A model for utilizing cloud computing in the smart grid domain. IEEE SmartGridComm'10, pages 483-488, 2010.

[11] Y. Simmhan, S. Aman, B. Cao, M. Giakkoupis, A. Kumbhare, Q. Zhou, D. Paul, C. Fern, A. Sharma, and V. Prasanna. An informatics approach to demand response optimization in smart grids. Technical Report, Computer Science Department, University of Southern California, 2011.

[12] Y. Simmhan, A. Kumbhare, B. Cao, and V. Prasanna. An analysis of security and privacy issues in smart grid software architectures on clouds. IEEE International Conference on Cloud Computing, 2011.
[13] C. Wang, K. Ren, and J. Wang. Secure and practical outsourcing of linear programming in cloud computing. In INFOCOM, 2011 Proceedings IEEE, pages $820-828,2011$.

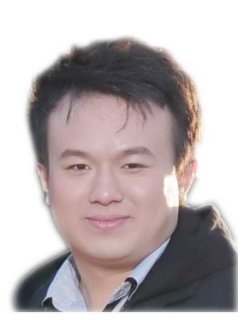

Xi Fang (Student Member 09) received the B.S. and M.S. degrees from Beijing University of Posts and Telecommunications, China, in 2005 and 2008 respectively. Currently he is a Ph.D student in the School of Computing, Informatics, and Decision Systems Engineering at Arizona State University. He has received a Best Paper Award at IEEE MASS'2011, a Best Paper Award at IEEE ICC'2011, and a Best Paper Award Runner-up at IEEE ICNP'2010. His research interests lie in wireless networks, smart grid, and cloud computing.

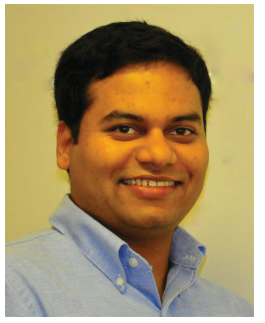

Satyajayant Misra (Student Member 2005, Member 2009) is an assistant professor at New Mexico State University. His research interests include anonymity, security, and survivability in wireless networks and protocol design for supercomputing and smart grid architectures. He serves on the editorial boards of the IEEE Communications on Surveys and Tutorials and the IEEE Wireless Communications Magazine, is the TPC Vice-Chair of Information Systems for IEEE INFOCOM 2012, and has served on the executive committees of IEEE SECON 2011 and IEEE IPCCC 2010. One of his co-authored papers was a runner-up to the best-paper award at IEEE ICNP 2010.

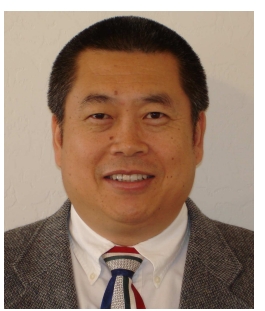

Guoliang Xue (Member 1996, Senior Member 1999, Fellow, 2011) is a Professor of Computer Science and Engineering at Arizona State University. He received the PhD degree (1991) in computer science from the University of Minnesota, Minneapolis, USA. His research interests include survivability, security, and resource allocation issues in networks ranging from optical networks to wireless mesh and sensor networks and social networks. He received Best Paper Awards at IEEE Globecom'2007, IEEE ICC'2011, IEEE MASS'2011, and IEEE Globecom'2011. He received the NSF Research Initiation Award in 1994, and has been continuously supported by federal agencies including NSF and ARO. He is an Associate Editor of IEEE/ACM Transactions on Networking and IEEE Network. His recent conference services include TPC co-chair of IEEE INFOCOM'2010. He was a Keynote Speaker at LCN'2011 and a Plenary Speaker at ICCCN'2011. He is a Distinguished Lecturer of the IEEE Communications Society. He is a Fellow of the IEEE.

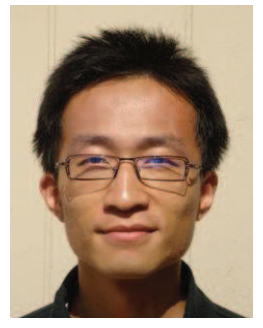

Dejun Yang (Student Member 08) received his B.S. from Peking University, Beijing, China, in 2007. Currently he is a $\mathrm{PhD}$ student in the School of Computing, Informatics, and Decision Systems Engineering (CIDSE) at Arizona State University. His research interests include economic and optimization approaches to wireless networks and social networks. He has received a Best Paper Award at IEEE ICC'2011, a Best Paper Award at IEEE MASS'2011 and a Best Paper Award Runner-up at IEEE ICNP'2010. 